\title{
Fish Two Ways: An Interdisciplinary Investigation of Biodiversity
}

\section{Robyn A. Puffenbarger and Kimberly J. Bolyard}

\author{
Bridgewater College, Department of Biology and Environmental Science, 402 E College St, \\ Bridgewater VA 22812-1599 USA \\ (rpuffenb@bridgewater.edu; kbolyard@bridgewater.edu)
}

\begin{abstract}
Identification at the species level is a challenge for both novice and experienced biologists. Traditionally, morphology is used for identification of specimens. It is challenging to use a dichotomous key based on morphological traits for species identification in organisms like vertebrate fishes. Since DNA sequencing is cheap and efficient, molecular information via DNA barcoding can help assist identification studies. For students, this is a multi-lab activity that works through two ways to identify fish species from local streams using morphology and DNA.
\end{abstract}

Keywords: biodiversity, morphology, dissection, DNA barcoding

Link to Supplemental Materials: https://doi.org/10.37590/able.v41.sup49

\section{Introduction}

Identification at the species level allows scientists to study many questions in ecology. Students can learn to identify rodent species in owl pellets, butterflies at pollinator gardens, birds in the forest, and the plants of an ecosystem with practice. Species level and accurate identification is the heart of many investigations including what species eat (Shehzad et al. 2012) and how to identify cryptic species (Burns et al. 2008).

Scientists have many reasons for precise identification of species. Biodiversity is decreasing at an alarming rate. Scientists who manage or conserve aquatic resources need information on what species are present or absent, current population densities, and community and ecosystem interactions.

In several field biology courses at Bridgewater College, students sample local streams and begin to learn fish identification. However, many species defy identification to species level, even from faculty with years of experience. Colloquially, you might hear 'minnow' to describe any small, silver freshwater fish. Saying 'minnow' is not precise, so we have applied DNA technology to these tricky identification questions.
DNA barcoding is used in the filed to search for and to identify new species (Pennisi 2019), to determine ecological distributions of fishes, taxonomically diverse vertebrates (Hubert et al. 2008), and to verify fish species in markets for regulatory compliance (Handy et al. 2010). This lab introduces students to this widely-used and powerful species identification tool by pairing it with the more familiar method of using morphological characters and a dichotomous key.

To use both parts of the laboratory exercises, a faculty member will need some familiarity with fish morphology, dissection, and use of a dichotomous key, plus a working knowledge of DNA extraction, PCR and gel electrophoresis. Since neither of us has both sets of expertise, we recommend a partnership where one professor tackles the morphology, and the other does the DNA. We also recommend crosstraining where each professor goes into the lab with the novice students to model life-long learning.

Depending on your course(s), discussions extending the activity might include evaluating systematic relationships among species, geographic variation in morphology among fishes, use of DNA barcoding in eDNA research, and environmental informatics. Overall, our activity emphasizes the importance of using interdisciplinary methods to identify species while examining biological diversity. 


\section{Student Outline}

\section{Part A: Morphology Lab}

\section{Objectives}

- Identify local freshwater fishes using morphological traits and a dichotomous key

\section{Methods and Data Collection}

In the Lab: Using Preserved Specimens, Learn the Morphological Features of Fishes and How to Use a Dichotomous Key

1. Work in pairs and identify the external morphological traits of your preserved specimens ( Fig. 1).

2. Complete Table 6.1 in Caillet et al. (1996).

3. Using a dichotomous key of freshwater fishes, identify specimens A, B, and C to family.

4. Using the key for each family identify the specimens to species level.

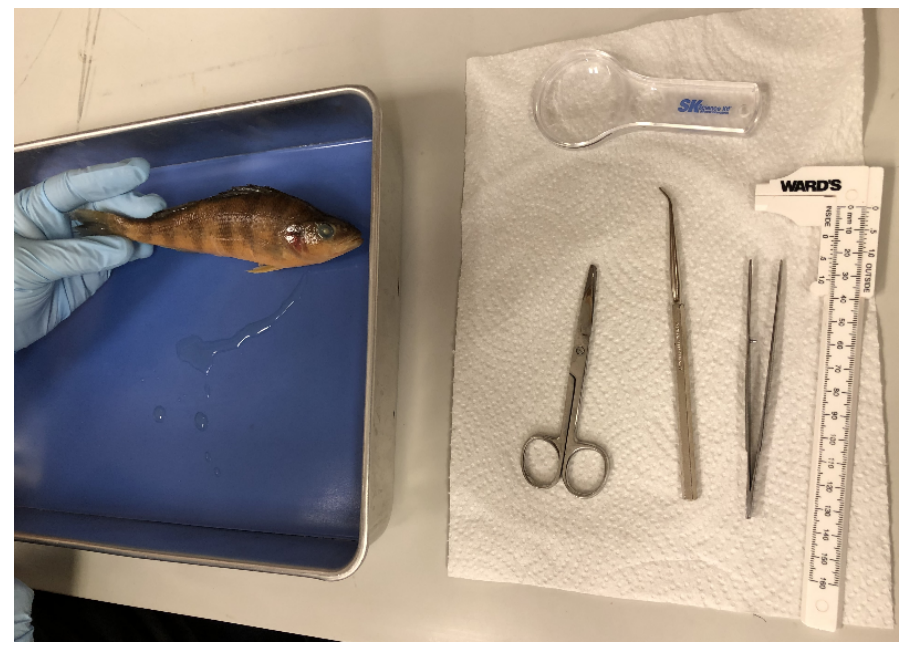

Figure 1. Preserved fish specimen on dissection tray with tools.

In the Field: Identify Local Fish Species using Morphological Traits.

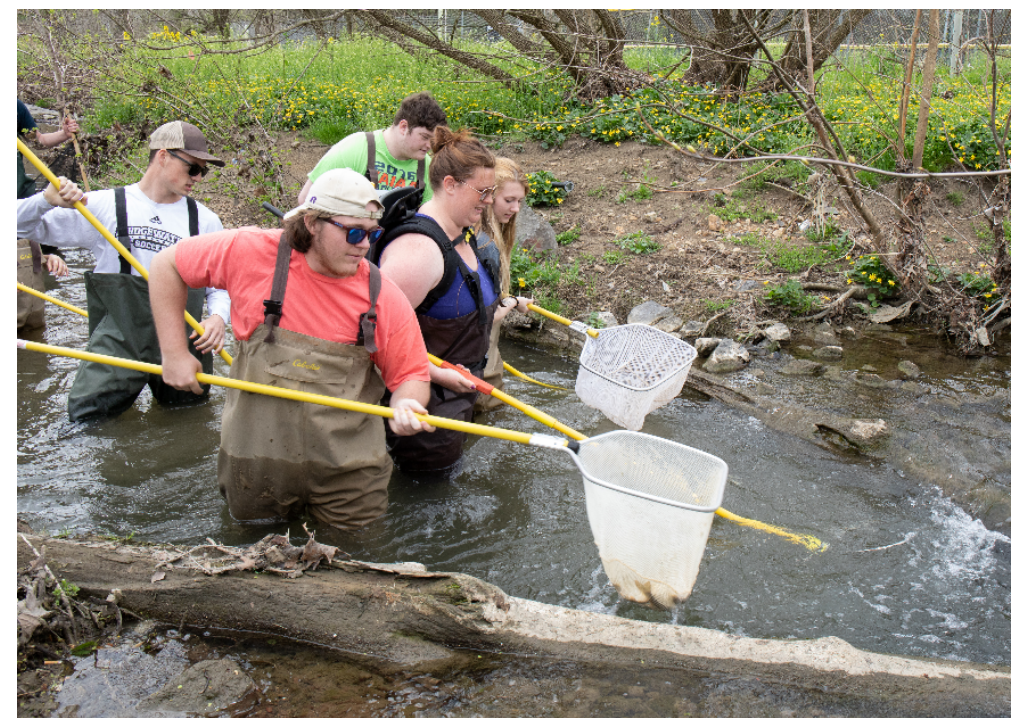

Figure 2. Students using electroshocker and nets to collect fish.
Collect Fish Tissue for DNA Barcoding

1. Using the seine net or backpack electroshocker, collect fishes in a small stream (Fig 2).

2. Identify fishes to the species level using morphological traits, keys, and identification guides (Fig 3).

3. Working with one fish at a time, anesthetize your fish in MS-222 (tricaine methanesulfonate).

4. Using clean scissors and forceps, remove a small piece of the posterior edge of the dorsal fin (approximately $1 \mathrm{~cm} \mathrm{x} 1 \mathrm{~cm}$ ) and place it into a small tube $(1.7 \mathrm{~mL}$ ) with $95 \%$ ethanol (Fig 4).

5. Place the fish into fresh, aerated stream water until it recovers. Return the fish to the stream. 


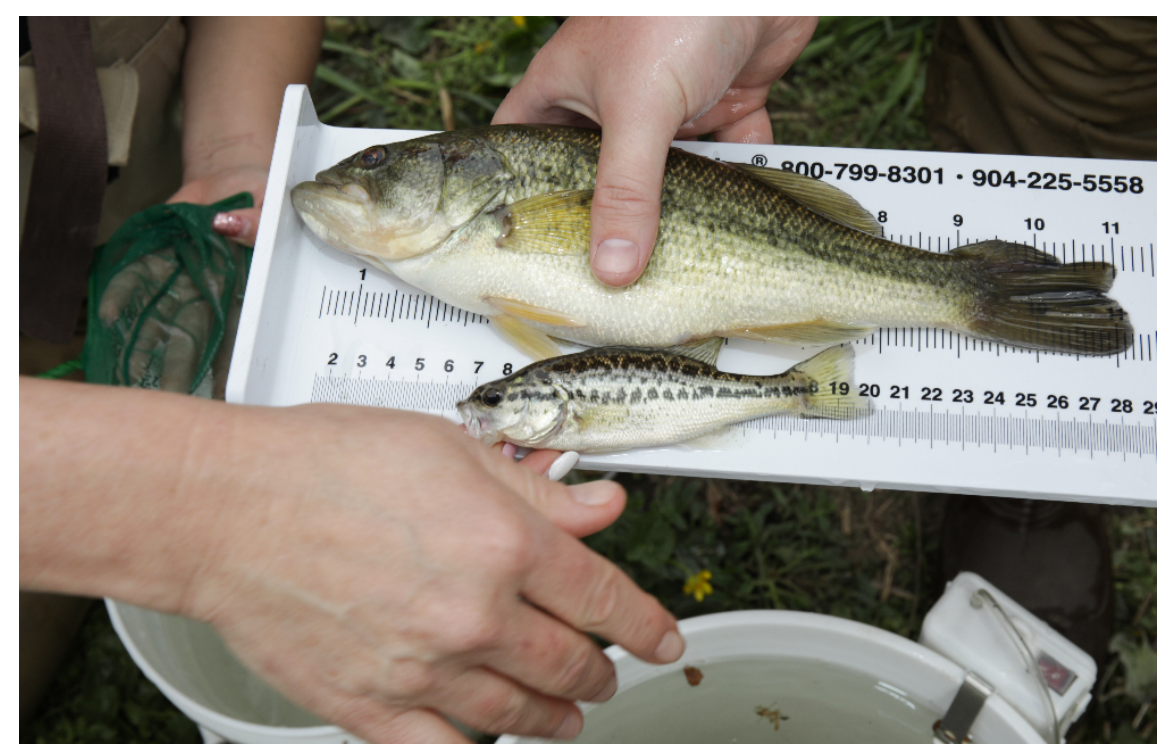

Figure 3. Measuring fish in the field before returning them to the stream.

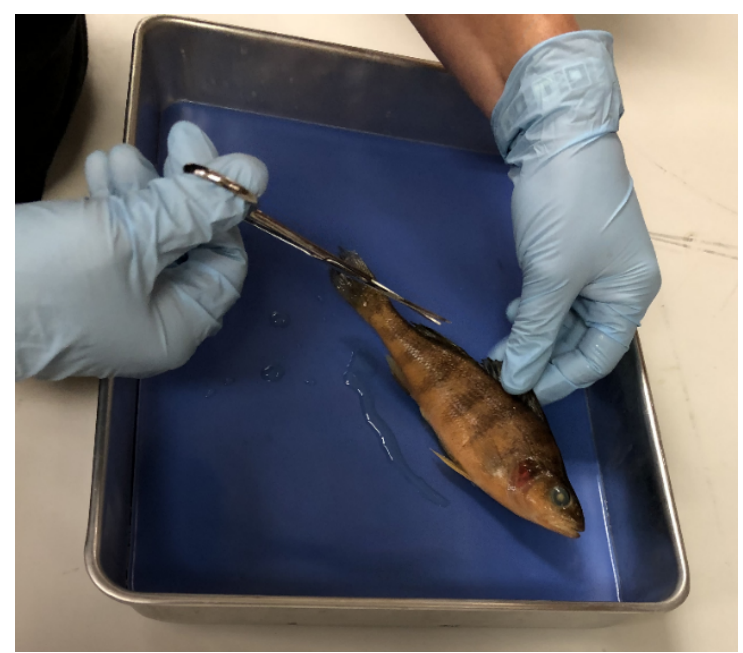

Figure 4. Preparing fish for fin removal. A small section of fin is removed for DNA isolation in the lab.

\section{Part B: DNA Barcoding Lab}

This lab demonstrates the use of PCR and DNA sequencing to generate a DNA Barcode used to identify environmental samples.

\section{Objectives}

Skills

You will begin developing:

- Manual dexterity in the use of pipets and small PCR

\section{Knowledge}

This lab starts building your:

- Understanding how to avoid contaminating samples

- Understanding how to isolate DNA for use in PCR

- How gel electrophoresis works to separate DNA by size 


\section{Introduction}

An explosion of quick and inexpensive methods to isolate, purify, amplify, and sequence DNA has brought new methods to help identify different species. Using DNA-based technologies, a multinational alliance of scientists is now cataloging life using a DNA barcoding system in order to accelerate the discovery of new species and develop powerful new tools to monitor and preserve Earth's vanishing biodiversity. In much the same way that a UPC (universal product code) barcode can differentiate a carton of milk from a bag of carrots when they are scanned into the cash register at a grocery store, DNA sequences can be used to uniquely identify different species. This is the basis of DNA barcoding.

We will work with the freshwater fishes of our local streams. While it is not always difficult to identify our local species, and colloquially, we can call anything of a certain size a minnow, our colleagues in biology must ID fish caught with much more certainty than that! In this year's lab, we will be doing identification of fish samples from a number of projects done by other biology students where fishes were caught via the electroshocker but were difficult to identify with certainty.

Note: PCR involves amplification of DNA and therefore it is critical to use proper technique to avoid any cross contamination between fish samples during DNA extraction. Do not recycle cutting implements, pipet tips, or containers. If using gloves, change gloves in between the handling of different fish samples!

\begin{tabular}{|c|l|c|}
\hline \multicolumn{2}{|c|}{ Table 1. Genes for the DNA barcodes across the kingdoms in domain Eukarya. } \\
\hline Kingdom & Barcode Genetic Target & DNA Source \\
\hline Animals & $\begin{array}{l}\text { Cytochrome c Oxidase Subunit I } \\
(C O I)\end{array}$ & Mitochondria \\
\hline Fungi & $\begin{array}{l}\text { Internal Transcribed Spacer in the } \\
\text { Ribosomal Cistron (ITS })\end{array}$ & Nucleus \\
\hline Plants & $\begin{array}{l}\text { Ribulose-1,5-bisphophate carboxylase } \\
(r b c L) ; \text { Maturase K }(\text { matK }) ; \text { non- } \\
\text { coding spacer region }(\text { trnH-psbA) }\end{array}$ & Chloroplasts \\
\hline from https://ibol.org/about/dna-barcoding/
\end{tabular}

Mitochondrial DNA is an ideal choice to serve as the barcode region for animals given that sequence differences among species are much more numerous within the mitochondrial genome than in nuclear DNA (Table 1). This is because mitochondrial DNA mutates at a faster rate than nuclear DNA. Additionally, the number of mitochondria can vary per cell type from just one mitochondrion to hundreds or thousands, whereas cells have only one nucleus. Thus, mitochondrial DNA is more abundant than nuclear DNA in any given tissue. This facilitates recovery of more copies of the target gene from every sample.

Within the mitochondrial genome, the COI gene encodes subunit 1 of the cytochrome c oxidase enzyme, and it is a portion of this gene that serves as the barcode region. Cytochrome $\mathrm{c}$ oxidase is an enzyme found in bacteria and in mitochondria. It is the final enzyme in the electron transport chain of cellular respiration, the process by which organisms harvest energy in the form of adenosine triphosphate (ATP) from food sources (Fig. 5). 


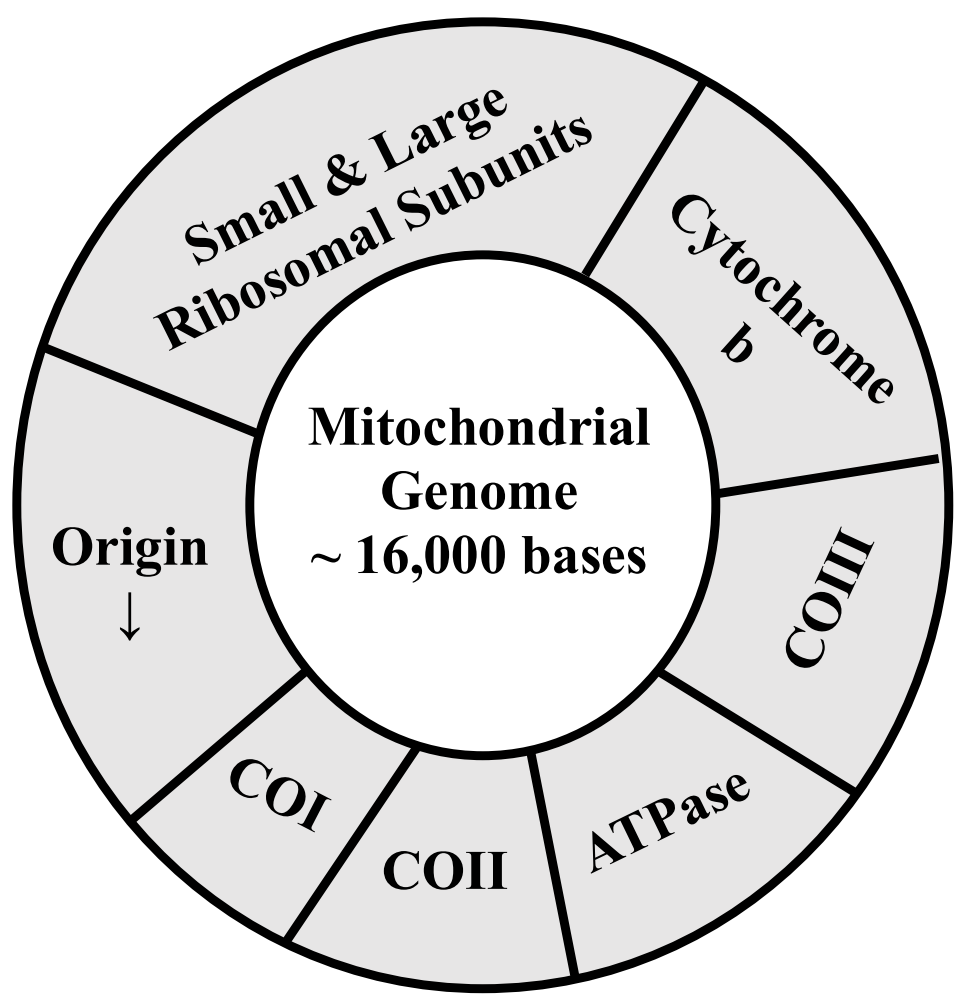

Figure 5. Schematic representation of some mitochondrial genes. The mitochondrial genome varies in size from species to species and it has crucial genes for the oxidative phosphorylation pathway to generate ATP. The cytochrome $c$ oxidase I gene (COI) used for DNA barcoding in most animal species is part of the mitochondrial genome.

Once you extract DNA from fish tissue, those samples of DNA and some additional experimental control samples will go into a polymerase chain reaction (PCR). PCR is DNA replication in a test tube. PCR allows you to amplify specific regions of DNA and make millions of copies of the target sequence. Your experiment in this lab is to make enough copies of the COI target sequence so that the resulting PCR product can be visualized by gel electrophoresis and then submitted for DNA sequencing.

For the PCR reactions, each fish DNA extracted, as well as two control PCR reactions were set up. One control PCR reaction will be the positive control, which will use pCOI plasmid DNA as your target sequence. The other reaction will be a negative control, which will use water instead of target DNA. Since water should not have any DNA in it, there should be no amplification of any target sequence in this sample. By having a known control that you are sure should not amplify the COI target sequence, you can tell if your PCR reactions have been contaminated by DNA containing the COI gene.

The lane corresponding to the positive control pCOI PCR should contain a $650 \mathrm{bp}$ band (Fig. 6). The presence of this band indicates whether a particular sample contained DNA that was amplifiable by COI gene-specific primers. If this lane does not contain a 650 bp band, it could be an indication that the PCR reactions were not assembled properly or that the thermal cycler was not functioning properly. The negative control should not contain a 650 bp band. If one is present, this indicates the PCR reaction(s) became contaminated at some point during processing and that your sequencing results may be negatively impacted due to the presence of additional contaminating DNA in your samples.

The lanes with your amplified fish DNA should contain a $650 \mathrm{bp}$ band that corresponds to the amplified COI gene, which you will go on to sequence. If there is no band present in some or all of these samples but the positive control COI PCR worked, this is an indication that something may have gone wrong during your DNA extraction procedure or that the type of fish tissue sample chosen was not optimal for this protocol. The species identity of the positive control DNA is Sebastes goodei, or chili-pepper rockfish. In some or all lanes containing PCR products you may notice a faint band around $100 \mathrm{bp}$ in size. This band corresponds to low level primer dimer formation during PCR. 


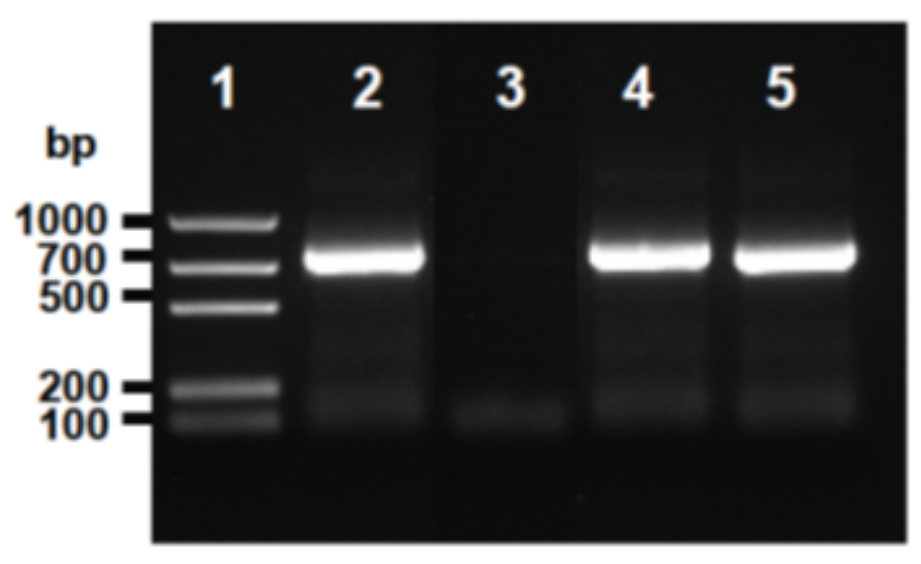

Figure 6. Fish DNA Barcode Gel Image. After gel electrophoresis of DNA samples, acquire an image of the gel(digital picture, imaging system, etc.). Lane 1 is the molecular weight markers supplied by BioRad to measure the DNA sizes in the gel. Lane 2 is the positive control sample also provided by BioRad in the kit. Lane 3 is a negative control and both lanes 4 and 5 show fish sample amplification. Samples with fish DNA, if amplification with PCR was successful should have a band of approximately 650 base pairs (bp). It is useful to run a negative control where fish DNA is replaced with water (lane 3) to show no amplification and the primer dimers that might form a low molecular weight haze at the 100-200 bp.

Once a DNA barcode is generated, in order understand what that DNA sequence means, it is necessary to have a searchable database that links the DNA barcodes generated by researchers from known and highly characterized biological specimens to the specimens' formal names and other important information (including data that allow the origin and current location of the source specimen to be easily tracked and verified by other researchers if necessary). Since DNA barcodes from unknown samples will be compared against those in the database, it is critical that a) the database contains sequence data of the highest quality possible, and b) that the identity given to the species from which the reference DNA barcode is generated is assigned correctly.

For this reason, scientists developed a highly regulated database called the Barcode of Life Data Systems (BOLD) reference library, which stores all the high-quality reference DNA barcode records. Through the BOLD Identification System (BOLD-IDS), a query (unknown or unverified) barcode sequence obtained from an unknown tissue sample or food product can be compared against reference barcode sequences contained in the BOLD reference library to determine the identity of the unknown specimen. The BOLD Systems website can be found at www.boldsystems.org.

To finish the DNA barcoding experiment, the PCR products must be sequenced to determine the linear order of bases GATC in the DNA. It should be noted that since DNA sequencing operates on the same principles of DNA replication that PCR does, any remaining unreacted reagents from PCR can interfere with the sequencing reactions. These reagents can include nucleotides, PCR primers, Taq polymerase, and buffer. Before running sequencing reactions, these reagents are removed using size exclusion chromatography. Size exclusion chromatography is a method of separation that allows very large molecules to run through a resin while very small molecules are retained (or vice versa). This means that the large PCR product can be collected while unreacted nucleotides, unreacted PCR primers, and Taq polymerase will remain on the column. Some sequencing facilities will perform these purifications for you while others require PCR products to be cleaned before they are submitted. In our case, the company that does our sequencing will do the post-PCR clean-up for us.

\section{Questions}

1. Draw the gel set up and label the wells, show the direction of DNA movement, and label where is the + and - to set up the current.

2. Where is DNA found in eukaryotic cells - HINT it is more than 1 place(!!!!)?

3. What parts of the cell must be broken down to extract DNA? 
4. It is important to keep track of the location of the DNA at each stage of purification. For the following steps of the protocol, state whether the DNA is in the pellet, in the supernatant, bound to the column, or in the flow through:

a. After centrifuging down the neutralized fish tissue lysate (pellet or supernatant).

b. After centrifuging the supernatant through the column (column or flow through).

c. After centrifuging the wash solution through the column (column or flow through).

d. After centrifuging the elution solution through the column (column or flow through).

5. Why is it important to use a new cutting utensil for every fish sample?

6. How do researchers target the portion of DNA to be amplified during PCR?

7. Do you expect the pCOI plasmid to generate a PCR product?

8. What about the negative control?

9. What is the molecular weight marker and is it a control?

\section{BLAST Sequence Analysis}
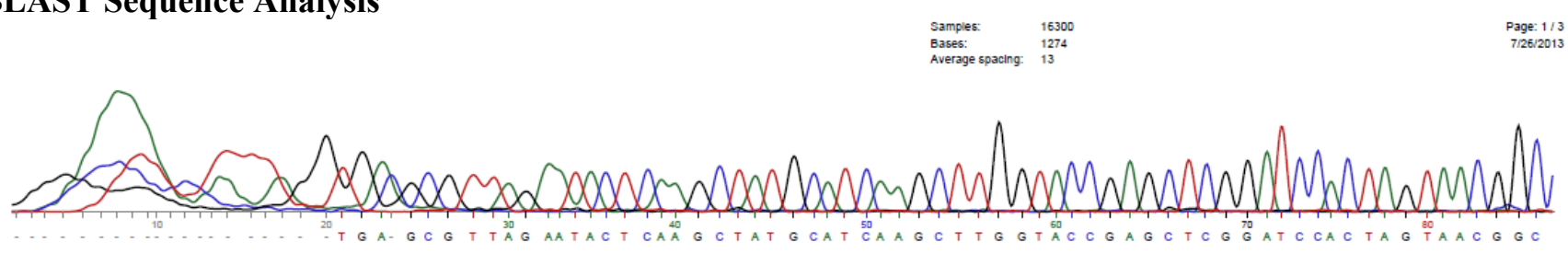

Figure 7. DNA trace from a sequence analysis. Each base gets a separate color. Very near to the sequencing primer, the run is often not sharp. As the reaction continues away from the primer start site, the sequence should give very sharp peaks telling us which base is present. At about base 40, there are two A's in a row, giving that 'mushy' double peak.

In DNA barcoding, a sample is selected, in our case, we are looking at fish collected from local streams. After getting the fish tissue, you isolated DNA. Then we took the DNA for PCR using primers to the COI sequence from the mitochondria that is the barcode area for vertebrates. After PCR, the samples were checked for amplification by gel electrophoresis and then sent to a company for sequencing.

Now it is time to see if the DNA sequence from the positive control is in one of the DNA databases!

1. Log on the web and go to this site: http://www.ncbi.nlm.nih.gov/BLAST/

2. In the section labeled Basic BLAST, click on: nucleotide BLAST

3. Enter the string of bases from the positive control into the big SEARCH box at the top of the page. Under Choose Search Set, select others as your database and then hit the BLAST! button.

4. Hit the format button and wait for your results to come up in the window. It should take about 30 seconds to a minute.

5. The top of the results window has some computer info about the program, scroll down to see a graph in lots of colors, keep scrolling and you get to a list with the header: "Sequences producing significant alignments".

6. Click on the match that has the highest match.

7. Write in the: 
a. accession number

b. source organism (common name and scientific name) - this tells you what organism the gene came from

c. definition-you are looking for the name of the gene, remember this PCR is for COI

d. The positive control was from the chili-pepper rockfish, Sebastes goodei, does the positive control match the BLAST search?

Now it is time to see if the DNA sequence from your PCR sample is in one of the DNA databases!

1. Log on the web and go to this site: http://www.ncbi.nlm.nih.gov/BLAST/

2. In the section labeled Basic BLAST, click on: nucleotide BLAST

3. Enter the string of bases from your group, with the FORWARD designation into the big SEARCH box at the top of the page. Under Choose Search Set, select others as your database and then hit the BLAST! button.

4. Hit the format button and wait for your results to come up in the window. It should take about 30 seconds to a minute.

5. The top of the results window has some computer info about the program, scroll down to see a graph in lots of colors, keep scrolling and you get to a list with the header: "Sequences producing significant alignments".

6. Click on the match that has the highest match.

7. Write in the code for the fish your group sampled:

From the BLAST search with the highest match, write in:

a. accession number

b. source organism (common name and scientific name) - this tells you what organism the gene came from

c. definition -- this is the name of the gene - looking for COI

Please redo the BLAST search with the REVERSE designation. Does it match what you wrote in above? If yes, super. If not, please fill in below!

a. accession number

b. source organism (common name and scientific name) - this tells you what organism the gene came from

c. definition -- this is the name of the gene - looking for COI

\section{Discussion Questions}

Compare your species identifications using the two different methods. Did they match? What are some advantages and disadvantages of using morphological traits to identify fish species? What are some advantages and disadvantages to using DNA barcoding to identify fish species? 


\section{Materials}

Lab sessions on morphological identification should have dissecting trays, forceps, blunt probe, calipers, gloves and hand lens or dissecting microscope to count scales. Laboratory instructors should guide students through the use of a dichotomous or other key as students often struggle the first time they attempt identification. Field equipment includes seine nets or an electroshocker with long-handled nets, and buckets or coolers.

For the DNA barcoding, we adapted the BioRad Fish Barcoding Kit (1665100EDU) and DNA sequencing kit (1665116EDU) for this lab. A significant portion of the student handout in this article is from BioRad materials. The kit is designed to identify fish at supermarkets or restaurants. It works perfectly fine for wild-caught, freshwater fish muscle or fin samples. Please note, we have not been able to use this kit for fish samples stored in formalin or formaldehyde!

In order to use the BioRad kit, you will need a basic DNA laboratory setup.W e have used disposable plastic bulb pipets instead of micropipettes, with disposable tips. As long as you can measure 200, 250, 500 and $100 \mu \mathrm{L}$ with some accuracy, this should work. The lab needs several more expensive pieces of equipment including microcentrifuge, gel electrophoresis rig and power supply and PCR machine. In terms of disposable supplies, you will need gloves, pipets (or tips), 1.7 and $2.0 \mu \mathrm{L}$ tubes, plus whatever tubes fit your PCR machine. If you use the BioRad kit, all of the consumables, including solutions and PCR components come with the purchase.

\section{Notes for the Instructor}

Faculty could organize these laboratory experiences for students in a number of ways. If faculty have the proper permits and equipment, students could work on morphological identification of fish from local waterways. Alternatively, prepared fish specimens are available from a number of companies including Carolina Biological (https:// www.carolina.com) and Biology Products (https:// biologyproducts.com/).

To do morphological characterizations, faculty will need access to identification guides and materials such as the Freshwater Fishes of Virginia (Jenkins and Burkhead 1993), The Diversity of Fishes: Biology, Evolution, and Ecology (Helfman et al. 2009), Fishes:A Field and Laboratory Manual on Their Structure, Identification and Natural History (Calliet et al. 1996), Laboratory Manual on General and Special Ichthyology (Marenkov 2018), Peterson
Field Guide to Freshwater Fishes (Page and Burr 2011) and other online resources (www.fishbase.se/Images/Glospic/ G_Fig13b6181_LatLin e.jpg; www.efish.fishwild.vt.edu/). We recommend starting in the lab with known species of preserved specimens such as yellow perch (Perca flavescens) and then moving onto fieldsampling if possible.

Identifying fish via DNA barcoding requires that tissue samples are fresh, frozen, preserved in alcohol but not in formaldehyde or formalin. The BioRad fish barcodin kit (biorad.com) that identifies fish species from supermarkets will also work for wild-caught fish. We have used tissues from both fish muscle and fins for identification, and fin samples can be removed while releasing the fish live back into its habitat.

Instructors should check with their campus Institutional Animal Care and Use Committee regarding required approvals for catching and using live animals.

Faculty will need to share the DNA sequences with students to analyze. Students can use the free software ChromasLite (https://technelysium.com.au/wp/chromas/) to examine the sequence trace before using BLAST (https:// blast.ncbi.nlm.nih.gov/Blast.cgi) to identify the sample hopefully to the same local fish species identified using morphology. Please note, sometimes the DNA will return a sequnce that BLAST will identify as Pseudomonas or other prokaryote. These are negative results. There is much more available on DNA barcoding from the University of Guelph at the educational portal (http://www.barcodinglife.org/index.php).

\section{Cited References}

Burns JM, Janzen DH, Hajbabaei M, Hallwachs W, Hebert PDN. 2008. DNA barcodes and cryptic species of skipper butterflies in the genus Perichares in Area de Conservacion Guanacaste, Costa Rica. Proceedings of the National Academies of Science. 105(17): 6350-6355.

Cailliet, G, Love M, Ebeling A. 1996. Fishes: A Field and Laboratory Manual on Their Structure, Identification and Natural History. Waveland Press Incorporated, Long Grove, Illinois.

Handy, SM, Deeds, JR, Ivanova, NV, Herbert, PDN Hanner, RH, Ormos, A, Weigt, LA, Moore, MM, Yancy, HF. 2010. A single-laboratory validated method for the generation of DNA barcodes for the identification of fish for regulatory compliance. Journal of AOAC International. 94(1):201-210. 
Helfman, GS, Collette BB, Facey DE, Bowen BW. 2009. The Diversity of Fishes: Biology, Evolution, and Ecology. Wiley-Blackwell, Chichester.

Hubert, N, Hanner, R, Holm, E, Mandrak, NE, Taylor, E, Burridge, M, Watkinson, D, Dumont, P, Curry, A, Bentzen, P, Zhang, J, April, J, Bernatchez, L. 2008. Identifying Canadian freshwater fishes through DNA Barcodes. PLOS One. 3(6): 2490.

https://doi.org/10.1371/journal.pone.0002490

Jenkins, RE. and Burkhead NM. 1993. Freshwater fishes of Virginia. American Fisheries Society, Bethesda, Maryland.

Marenkov O. 2018. Laboratory Manual on General and Special Ichthyology. World News of Natural Sciences. 18(1): 1-51.

Pennisi, E. 2019. DNA barcodes jump-start search for new species. Science. 364:920-921.

Shehzad W, Riaz T, Nawaz MA, Miquel C, Poillot C, Shah SA, Pompanon F, Coissac E, Taberlet P. (2012). Carnivore diet analysis based on nextgeneration sequencing: application to the leopard cat (Prionailurus bengalensis) in Pakistan. Molecular Ecology. 21: 1951-1965.

\section{Acknowledgments}

Thank you very much to our faculty colleagues and students at Bridgewater College for your assistance over the many semesters testing these lab materials. We appreciate the opportunity to present at ABLE and the feedback from our workshop attendees.

\section{About the Authors}

Robyn Puffenbarger joined the faculty at Bridgewater College in 2001 and Kimberly Bolyard joined in 2007. Both are associate professors in the Department of Biology and Environmental Science. 


\section{Appendix A: DNA Sequences}

These are actual DNA sequences from the positive control provided by BioRad and two wild caught fish samples from local freshwater streams near Bridgewater Virginia. These sequences can be used in the BLAST protocol if you are not able to generate your own DNA samples. Using BLAST, both Fish \#2 fin and muscle will return the same species of fish.

Positive Control

CTAGTATTTGGTGCCTGAGCCGGTATAGTAGGCACAGCCCTCAGCCTACTCATTCGAGCAGAACTAAGCCAAC CGGGCGCTCTCCTTGGAGACGACCAAATTTATAATGTAATCGTTACAGCACATGCCTTCGTAATGATTTTCTTT ATAGTAATGCCAATTATAATTGGAGGTTTTGGAAACTGATTAATTCCCCTAATGATTGGAGCCCCAGATATAGC ATTTCCTCGTATAAATAACATAAGTTTCTGACTTCTTCCCCCTTCTTTCCTACTACTACTTGCCTCTTCTGGAGTA GAAGCAGGTGCCGGAACCGGGTGAACAGTGTACCCGCCCCTGGCCGGTAATTTAGCCCACGCAGGAGCATCAG TTGACCTAACAATCTTTTCACTTCACCTAGCAGGTATTTCCTCAATCCTCGGGGCAATCAATTTTATTACCACAA TTATTAATATGAAGCCCCCTGCCATCTCTCAGTACCAGACGCCCCTATTTGTATGAGCCGTCCTAATTACCGCC GTTCTTCTCCTTCTCTCTCTA

Fish \#2 Fin

AGCCTCCTAATTCGAGCCGAACTAAGTCAACCAGGCTCACTTCTAGGTGACGACCAAATCTATAATGTTATTGT TACTGCTCACGCCTTTGTAATGATTTTCTTTATAGTAATGCCAATTCTAATTGGTGGGTTCGGAAACTGACTTGT ACCTTTAATAATTGGAGCACCTGACATAGCATTTCCACGAATAAACAACATAAGCTTCTGACTCTTACCCCCGT CATTCCTACTGCTACTAGCCTCTTCTGGTGTTGAAGCCGGGGCCGGAACAGGATGAACTGTTTACCCCCCACTT GCAGGTAACCTTGCCCATGCAGGAGCATCAGTAGACCTTACGATCTTCTCTCTGCATTTAGCAGGTGTGTCATC AATTCTAGGGGCAGTTAATTTTATTACTACAATTATTAATATAAAACCCCCAGCAATCTCACAGTATCAAACAC CTCTCTTTGTGTGAGCCGTGCTTGTAACTGCCGTA

Fish \#2 Muscle

TTTAAGCCCTCCTAATTCGAGCCGAACTAAGTCAACCAGGCTCACTTCTAGGTGACGACCAAATCTATAATGTT ATTGTTACTGCTCACGCCTTTGTAATGATTTTCTTTATAGTAATGCCAATTCTAATTGGTGGGTTCGGAAACTGA CTTGTACCTTTAATAATTGGAGCACCTGACATAGCATTTCCACGAATAAACAACATAAGCTTCTGACTCTTACC CCCGTCATTCCTACTGCTACTAGCCTCTTCTGGTGTTGAAGCCGGGGCCGGAACAGGATGAACTGTTTACCCCC CACTTGCAGGTAACCTTGCCCATGCAGGAGCATCAGTAGACCTTACGATCTTCTCTCTGCATTTAGCAGGTGTG TCATCAATTCTAGGGGCAGTTAATTTTATTACTACAATTATTAATATAAAACCCCCAGCAATCTCACAGTATCA AACACCTCTCTTTGTGTGAGCCGTGCTTGTAACTGCCGTACTTCTACTCCTTTCACTACCCGTTCTAGCTGCCGG CATTACTATGCTTCTAACTG 
Appendix B: Gel Electrophoresis Results

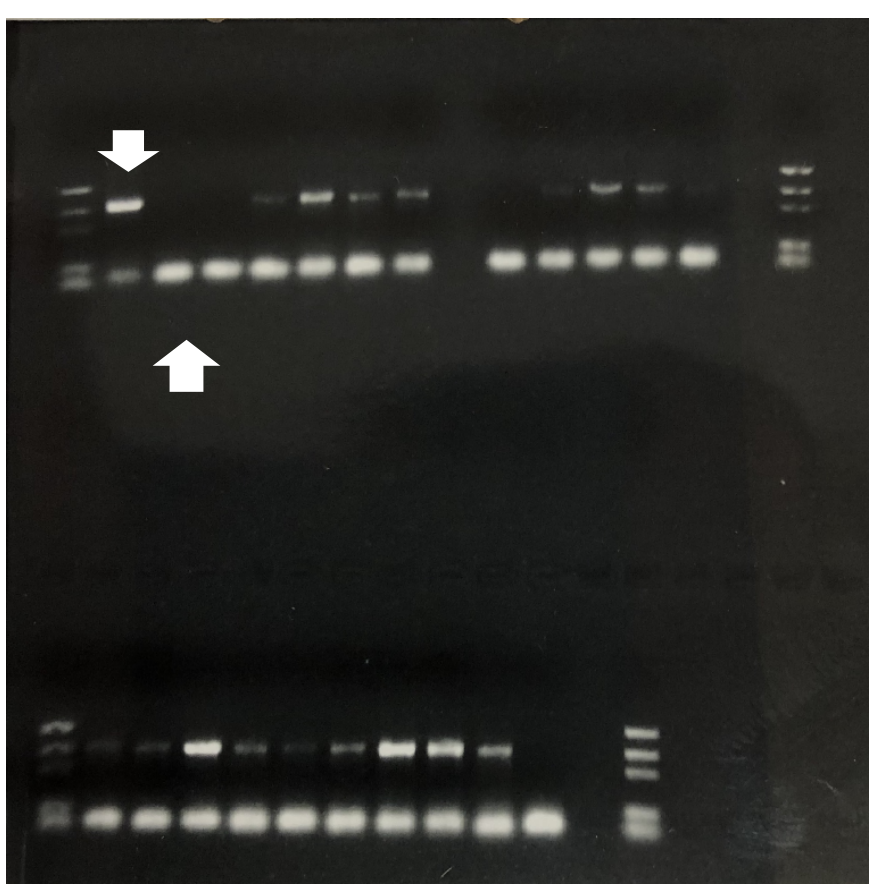

Figure 8. DNA bands from typical gel electrophoresis of wild-caught fish samples. Lanes with five bands are molecular weight markers, for example Lane 1 at top left. These bands will match the size of the BioRad kit shown in the student outline (1000 bp, 700 bp, 500 bp, 200 bp, $100 \mathrm{bp}$ ). Lane 2 at top shows clearly positive fish sample appropriate for sequencing (marked with arrow pointing down). The smaller molecular weight band is 'primer dimer,' an artefact of PCR. This artefact shows up even in negative controls, like Lane 3 (marked with arrow pointing up). 


\section{Mission, Review Process \& Disclaimer}

The Association for Biology Laboratory Education (ABLE) was founded in 1979 to promote information exchange among university and college educators actively concerned with teaching biology in a laboratory setting. The focus of ABLE is to improve the undergraduate biology laboratory experience by promoting the development and dissemination of interesting, innovative, and reliable laboratory exercises. For more information about ABLE, please visit http://www.ableweb.org/。

Advances in Biology lLaboratory Education is the peer-reviewed publication of the conference of the Association for Biology Laboratory Education. Published articles and extended abstracts are evaluated and selected by a committee prior to presentation at the conference, peer-reviewed by participants at the conference, and edited by members of the ABLE Editorial Board. Published abstracts are evaluated and selected by a committee prior to presentation at the conference.

\section{Citing This Article}

Puffenbarger RA, Bolyard KJ. 2020. Fish two ways: an interdisciplinary investigation of fish diversity. Article 49 In: McMahon K, editor. Advances in biology laboratory education. Volume 41. Publication of the 41st Conference of the Association for Biology Laboratory Education (ABLE). https://doi.org/10.37590/able.v41.art49

Compilation (C) 2020 by the Association for Biology Laboratory Education, ISBN 1-890444-17-0. All rights reserved. No part of this publication may be reproduced, stored in a retrieval system, or transmitted, in any form or by any means, electronic, mechanical, photocopying, recording, or otherwise, without the prior written permission of the copyright owner.

ABLE strongly encourages individuals to use the exercises in this volume in their teaching program. If this exercise is used solely at one's own institution with no intent for profit, it is excluded from the preceding copyright restriction, unless otherwise noted on the copyright notice of the individual chapter in this volume. Proper credit to this publication must be included in your laboratory outline for each use; a sample citation is given above. 